\title{
Early predictors of injectable disease modifying drugs suboptimal response based on clinical and radiological data assessment in Polish Multiple Sclerosis patients
}

\author{
Sławomir Wawrzyniak ${ }^{1}$, Dorota Koziarska ${ }^{2}$, Alina Kułakowska ${ }^{3}$, \\ Halina Bartosik-Psujek ${ }^{4}$, Monika Adamczyk-Sowa ${ }^{5}$ \\ ${ }^{1}$ Department of Neurology, Military Clinical Hospital No. 10 with Policlinic, Bydgoszcz, Poland \\ ${ }^{2}$ Department of Neurology, Pomeranian Medical University, Szczecin, Poland \\ ${ }^{3}$ Department of Neurology, Medical University of Bialystok, Bialystok, Poland \\ ${ }^{4}$ Department of Neurology, Faculty of Medicine, University of Rzeszow, Rzeszow, Poland \\ ${ }^{5}$ Department of Neurology, Medical University of Silesia, Zabrze, Poland
}

\section{ABSTRACT}

Background. Prospective database studies can provide useful information regarding 'real-world' outcomes and drug efficacy.

Objective. To determine the early predictors of suboptimal treatment responses at two and three years under injectable Disease Modifying Therapy (DMT).

Methods. This was a multi-centre prospective database study. Adult patients who started injectable DMTs between January 2008 and June 2013 were included. The follow-up continued until July 2014. Suboptimal treatment responses were defined as: the presence of clinical relapse and/or Expanded Disability Status Score (EDSS) progression and/or newly emerging T2 lesions or/and gadolinium enhancing lesions on magnetic resonance imaging (MRI). The parameters were assessed up to 24 months prior to, and every 12 months during, the treatment.

Results. Analysis included 297 MS (multiple sclerosis) patients followed for a mean time of $2.3 \pm 1.3$ years (range 1-5). Within the three years of observation, the persistence and efficacy with injectable DMTs was high. With increased disability, defined by EDSS $\geq 3$, the risk of treatment failure increased up to seven times, OR 7.33 in the second year radiological analysis ( $\mathrm{Cl} 95 \%$ : 1.69-29.2) $\mathrm{p}<0.01$, similar to over two times in the second year clinical analysis, with the baseline symptomatic hemiparesis OR 2.75 ( $C$ I 95\% : 1.06-7.06) p 0.034. A high relapse rate one year prior to treatment adversely influenced the treatment success at three years, OR 3.04 (CI 95\% : 1.49-8.43) $p<0.01$.

Conclusions . Injectable DMTs should not be chosen for treatment initiation in motoric disabled patients (EDSS $\geq 3$ ) with a high grade of clinical activity. These drugs are effective in less active relapsing-remitting (RR) MS patients.

Key words: multiple sclerosis, disease modifying treatment, annualised relapse rate, Expanded Disability Status Score, magnetic resonance imaging, RIO score, efficacy

(Neurol Neurochir Pol 2019; 53 (2): 131-137)

\section{Introduction}

Multiple sclerosis (MS) is a chronic demyelinating disease with increasing prevalence in Poland [1]. The course of the disease and its associated rate of disability progression are highly unpredictable and constitute the most common primary neurological cause of disability in young adults [2]. The continued development of diagnostic criteria and increased awareness of typical clinical features have allowed shortening of the diagnosis delay and an earlier

Address for correspondence: Dorota Koziarska, Department of Neurology, Pomeranian Medical University, 71-252 Szczecin, Unii Lubelskiej 1 Str., Poland, email: dorkoz@pum.edu.pl 
initiation of treatment [3]. An analysis of the disease onset symptoms and initial relapse rate can help to estimate the most probable course of the disease, with specification as to its aggressiveness [4]. However, the question as to which drug should be chosen for the treatment is still unresolved. The country-wide standardised inclusion and reassessment criteria allowed us to make joint analyses of patients from different centres at a time when only injectable disease modifying treatments (DMT) were available in Poland. First line injectable treatment options included different formulations of interferon beta (INT- $\beta$ ): subcutaneous IFN- $\beta-1 b$, IFN- $\beta-1 \mathrm{a}$ and intramuscular IFN- $\beta$ - $1 \mathrm{a}$ or subcutaneous glatiramer acetate (GA). These are well established safety immunomodulatory drugs with all-over efficacy defined in randomised trials at about 30\% [5-8]. This includes a moderate influence on lowering the relapse rate and on the clinical and radiological progression of disability. At a time when there are a growing number of newly available treatment options, it is still important to analyse previously used algorithms, either to reestablish them or to ensure correct treatment decisions [9].

\section{Material and methods}

\section{Patients}

The research design was a multi-centre prospective database study. Four medical centres (Bydgoszcz, Szczecin, Białystok, Zabrze) providing immunomodulatory treatment of MS within the nationally funded treatment programme participated in the research.

Inclusion criteria: adult (over 18 years old) patients with relapsing-remitting (RR) MS who started an injectable DMT between January 2008 and June 2013 were included in this study. The follow-up continued to July 2014 . All participants met the $2005 \mathrm{McD}$ nald criteria for MS and had either two relapses or one relapse and a chronologically (three months) separate gadolinium enhancing lesion on magnetic resonance imaging (MRI), within the immediate two year period preceding the start of DMT [3]. We included either patients with a newly confirmed diagnosis or untreated for at least the last six months with a new exacerbation and an Expanded Disability Status Score (EDSS) of up to 4.5. During the time of data collection (between 2008 and 2011), the treatment duration regime in Poland was limited to three years. Some active patients could be recruited several times, always after a new instance of disease exacerbation. Treatment selection for IFN $\beta$ or GA was based on national eligibility criteria. Patients with Clinical Isolated Syndrome (CIS) or progressive forms of MS were not eligible for this study.

\section{Ethics}

The work described in this article has been carried out in accordance with The Code of Ethics of the World Medical
Association (Declaration of Helsinki) for experiments involving humans; we have followed uniform requirements for manuscripts submitted to biomedical journals.

\section{Cohorts}

The time of treatment initiation and a follow-up period of a minimum of two years allowed us to make a comparative observation of two cohorts. Cohort A comprised patients included from January 2008 up to May 2012, eligible after at least two relapses during the last 24 months before qualification to the treatment. Cohort B comprised patients included from June 2012, eligible after at least one relapse during the last 12 months before qualification to the treatment.

\section{Method}

Patient data was collected in an electronic case report form. Clinical and demographic parameters were analysed. The disease activity was determined by the annualised relapse rate (ARR) defined as: MS-related hospitalisation, MS-related Emergency Room visit or MS-related outpatient visit with corticosteroid prescription \pm 7 days within one year. EDSS scoring was performed every three months during the treatment by trained staff [10]. Clinical neurological progression was defined as a change of EDSS score of more than 1.0 that was persistent for longer than three months despite a lack of relapse. Magnetic resonance imaging (MRI) was performed at the beginning and after each year of treatment, and was focused on detecting new emerging T2 lesions or/and a gadolinium (GD) enhancing lesion.

Outcomes were defined on the basis of the RIO score:

Optimal clinical response is described as no relapses, no progression in disability (defined as stable EDSS or with a change of less than 1.0) within a year. Optimal radiological response is defined as no changes in NMR (no gadolinium enhancing lesion and less than two T2 lesions). The definition is similar to a RIO score of zero. Suboptimal clinical response is described as one or more relapse and/or progression in disability (defined as increase of EDSS $\geq 1.0$ ) and/or suboptimal radiological response, defined as $>1$ active gadolinium enhancing lesion and/or $>2 \mathrm{~T} 2$ lesions in a year. This definition is similar to a RIO score of 1 or more [11].

\section{Statistics}

Statistical analysis was performed using $\mathrm{R}$ (The $\mathrm{R}$ Project for Statistical Computing)- $\mathrm{R}$ version 3.4.2. In descriptive statistics, the following tests were used: Fisher's exact test to establish differences in proportions, T-test to establish differences in quantitative data (for normally distributed data), Wilcoxon test to establish differences in quantitative data (for non-normally distributed data), Anderson-Darling test to establish the normality of given quantitative data. In order to 
determine the influence of main neurological rates (symptoms on MS onset, EDSS) on drugs suboptimal response, univariable logistic regressions were performed.

\section{Results}

Two hundred and ninety seven MS patients met the inclusion criteria. The female to male ratio was $2.3: 1$. The average age of the patients was $35.37 \pm 9.92$ years (range 18-64 years), and the patients showed mild disability characterised by a mean baseline EDSS of $1.95 \pm 1.05$. The average treatment delay was $4.12 \pm 4.81$ years (range $0-24$ years). The two studied cohorts were comparable without significant differences on baseline, except for the fact that the more recently included patients (Cohort B) were older, more disabled and more active in their disease course ( $\mathrm{p}$ 0.01). In both cohorts, the proportion between the newly diagnosed-naive and previously treated patients was similar (Tab. 1).

\section{Clinical outcome with injectable DMT}

Over $85 \%$ of patients were under INF $\beta$ treatment with a mean follow-up time of $2.3 \pm 1.3$ years (range 1-5). After three years of treatment, no relapses and stable EDSS was observed in 91 patients (85\%) which constitutes about one third of the baseline population. The patient loss in follow-up was mainly due to a treatment time limited to three years, established by the nationally funded treatment programme. The evidence of suboptimal treatment response defined as evidence of clinical and/or radiological activity, was stable in the follow up-period, within the range of $15-18 \%$. The reason for stopping or switching the initial DMT therapy was mainly driven by the instability of the clinical course. The mean EDSS score was stable over three years of treatment at about 2.0; we also observed a significant and stable reduction of ARR and GD enhancement $\mathrm{p}<0.01$ (Tab. 2).

\section{Main predictors of suboptimal response to injectable DMT in three years follow-up}

Suboptimal clinical response, defined as a RIO score $\geq 1$, was further analysed in two separate categories: clinical and radiological response. In univariable logistic regression, the factors predicting worse response to injectable DMTs were comparable in the whole three years of the follow-up period. The main predictor of inefficacy in the first and second years of treatment was the baseline clinical status. With an increase in disability defined as baseline EDSS $\geq 3$, the risk of treatment failure increased to over seven times (in the second year radiological analysis), similar to over two times with baseline symptomatic hemiparesis, which in fact influences the EDSS

Table 1. Socio-demographic and clinical characteristics of MS patients at baseline

\begin{tabular}{|c|c|c|c|c|}
\hline Variable & $\begin{array}{c}\text { Overall } \\
(297)\end{array}$ & $\begin{array}{l}\text { Cohort A } \\
(n=150)\end{array}$ & $\begin{array}{l}\text { Cohort B } \\
(n=147)\end{array}$ & p value \\
\hline Age (years) mean (SD) & $35.37(9.92)$ & $33.86(9.42)$ & $36.92(10.2)$ & $0.01^{* *}$ \\
\hline Gender (female/male), $n$ & $206 / 91$ & $100 / 50$ & $106 / 41$ & 0.38 \\
\hline Age at diagnosis (years) mean (SD) & $31.26(10.12)$ & $29.94(9.38)$ & $32.60(10.69)$ & 0.06 \\
\hline \multirow[t]{2}{*}{ Treatment delay (years) mean (SD) range } & $4.12(4.81)$ & $3.92(4.64)$ & $4.32(4.98)$ & 0.8 \\
\hline & $(0-24)$ & $(0-23)$ & $(0-24)$ & \\
\hline Previous DMT treatment (No/Yes), n & $212 / 85$ & $107 / 43$ & $105 / 42$ & 1.0 \\
\hline BL treatment INF/GA, $n$ & $253 / 44$ & $133 / 17$ & $120 / 27$ & 0.1 \\
\hline \multicolumn{5}{|l|}{ Onset symptoms: $\mathrm{n} / \%$} \\
\hline Optic neuritis: $\mathrm{n} / \%$ & $99 / 33.4$ & $52 / 33.8$ & $47 / 31.5$ & 0.71 \\
\hline Hemiparesis: $\mathrm{n} / \%$ & $77 / 25.9$ & $42 / 28.0$ & $35 / 24.2$ & 0.43 \\
\hline Cerebellar syndrome: $\mathrm{n} / \%$ & $35 / 11.8$ & $19 / 12.6$ & $17 / 11.6$ & 1.0 \\
\hline Brainstem syndrome: $\mathrm{n} / \%$ & $31 / 10.4$ & $16 / 10.6$ & $15 / 10.2$ & 1.0 \\
\hline Other: $\mathrm{n} / \%$ & $55 / 18.5$ & $21 / 14.0$ & $33 / 22.5$ & 0.2 \\
\hline EDSS on BL mean (SD) & $1.95(1.05)$ & $1.75(0.97)$ & $2.15(1.10)$ & $0.01^{*}$ \\
\hline $\mathrm{EDSS} \geq 3$ on $\mathrm{BL} n / \%$ & $44 / 14.81$ & $16 / 10.66$ & 28/19.04 & $0.05^{*}$ \\
\hline NMR GD enhancement on BL & $62 / 297$ & $18 / 132$ & $44 / 103$ & $<0.01^{* *}$ \\
\hline ARR 2 years before BL (SD) & $1.535(0.95)$ & $1.37(0.86)$ & $1.70(0.96)$ & $0.01^{*}$ \\
\hline ARR 1 year before BL (SD) & $1.18(0.78)$ & $1.0(0.68)$ & $1.36(0.89)$ & $0.01^{*}$ \\
\hline ARR 6 months before BL (SD) & $0.89(0.68)$ & $0.71(0.56)$ & $1.07(0.81)$ & $0.01^{*}$ \\
\hline
\end{tabular}

p value represents the statistical difference between the two cohorts; *Wilcoxon test; **Fisher test; ARR — annual relapse rate; BL — baseline; DMT — disease modifying therapy; EDSS — Expanded Disability Status Score; MRI — magnetic resonance imaging; SD — standard deviation 
Table 2. Clinical characteristics of MS patients during treatment

\begin{tabular}{|c|c|c|c|c|c|}
\hline Variable & BL & I year & Il year & III year & p value \\
\hline Overall patients, $\mathrm{n}$ & 297 & 297 & 167 & 107 & - \\
\hline EDSS mean (SD) & $1.95(1.05)$ & $1.96(1.12)$ & $1.96(1.12)$ & 2.018 & 0.84 \\
\hline $\operatorname{ARR}(S D)$ & $1.53(0.95)$ & $0.13(0.41)$ & $0.13(0.41)$ & $0.13(0.41)$ & $<0.01$ \\
\hline NMR GD enhancement mean (SD) & $0.54(1.42)$ & $0.06(0.32)$ & $0.06(0.32)$ & $0.06(0.32)$ & $<0.01$ \\
\hline Continued treatment $\mathrm{n} / \%$ & - & $283 / 95.0$ & $161 / 96.4$ & $104 / 97.2$ & 0.43 \\
\hline $\begin{array}{l}\text { Optimal clinical/radiological response } \\
(\text { RIO 0) n/\% }\end{array}$ & - & $244 / 82.2$ & $142 / 85.0$ & $91 / 85.0$ & 0.52 \\
\hline Suboptimal clinical/radiological response $(\mathrm{RIO} \geq 1) \mathrm{n} / \%$ & - & $53 / 17.8$ & $25 / 15.0$ & $16 / 15.0$ & 0.51 \\
\hline
\end{tabular}

p value represents the statistical difference between the analysed groups; ARR — Annual Relapse Rate; BL — baseline; EDSS — Expanded Disability Status Score; GD — gadolinium; SD — standard deviation

Table 3. Main predictors of suboptimal response to injectable DMT in three years follow-up: univariable logistic regression

\begin{tabular}{lccc} 
Suboptimal response & Variable & OR & Cl 95\% \\
\hline First year clinical & EDSS on BL & 1.38 & $1.00-1.891$ \\
& Hemiparesis on BL & 2.17 & $1.04-4.406$ \\
First year radiological & EDSS on BL & 0.55 & 0.048 \\
& Age at treatment initiation & 0.93 & 0.034 \\
Second year clinical & Hemiparesis on BL & 2.75 & $0.88-0.98$ \\
Second year radiological & EDSS on BL & 2.13 & $1.065-7.066$ \\
& EDSS on BL $\geq 3$ & 7.33 & $1.143-4.072$ \\
Third year clinical & ARR one year prior to treatment & 3.047 & $1.69-29.206$ \\
Third year radiological & ARR one year prior to treatment & 3.667 & $1.322-7.71$ \\
\end{tabular}

ARR — Annual Relapse Rate; BL — baseline; EDSS — Expanded Disability Status Score

score. The younger age and lower EDSS on treatment initiation positively influenced optimal radiological response in the first year. With longer treatment duration, the ARR one year before the treatment initiation seems to play a negative role. With a higher relapse rate, the suboptimal response risk increased three times in the third year of treatment. No other demographic or clinical factors played a role in predicting a worse outcome (Tab. 3)

\section{Discussion}

Open compartmental studies concerning first-line RRMS treatment have shown a significant reduction of disease activity. In the first two years of INF B treatment, the ongoing disease activity has been reported as between 16\% and 29\% [12-16]. Freedom from disease activity has become a widely proposed system of optimal clinical outcomes analysis based on relapse rate, EDSS and radiological signs of new active lesions. The often used RIO score can be translated to NEDA showing an optimal disease control in a similar way [16-18]. In this multi-centre prospective database study, we showed a high efficacy of injectable first-line DMTs. This was achieved by a good clinical response in lowering the BL rate of ARR from 1.3 to 0.13 (after one and two and three years of treatment). EDSS stabilization, together with the reduction of radiological progression, resulted in good disease control in over $80 \%$ of patients.

Some other studies, in so far as our results, show high efficacy of first-line injectable medications in the treatment of RRMS patients [18-23]. In the study by Sorenson et al., based on the Danish Nationwide Database containing health records from RRMS patients, there was no clear difference in the efficacy of immunomodulatory treatment with injectable DMTs [22, 24-25].

We based the comparison of the optimal and suboptimal treatment responses on the RIO score. To analyse the predictors for suboptimal response, we concentrated mainly on baseline variables that could lead to treatment failure. Baseline EDSS $\geq 3$ or hemiparesis as the initial MS manifestation increases such a risk more than two times. A similar conclusion without any specific cut-off was described in an 18 year-long observational cohort study of treatment-naïve patients from Canada [19]. Also in other reports, high EDSS score has been considered to be the main factor predicting interruption of 
therapy, which in fact is similar to a suboptimal treatment response [26-27]. Due to the therapy outcome optimisation, it is recommended attention be paid to more active/progressive patients at diagnosis [4]. Those patients with higher levels of disability in the early relapsing-remitting course of the disease should be considered as highly active, with an indication for therapeutic decisions other than first line DMTs [9]. The same is true for relapse active patients before the treatment, as relapses are the clinical manifestation of inflammation. In our cohort, the risk of being suboptimal responders at three years was more than three times higher for relapse active patients one year before treatment.

Another important aspect that we took into consideration was treatment efficacy estimation by early and late therapy initiation. We could compare the outcomes form treatment-naive and retreated patients. This observation was possible because of country-specific regulation on the time of data collection. According to the previously used (from 2008 to 2012) guidance, the nationally funded treatment programme was limited to three years. Some active patients could be recruited several times, always after a new exacerbation of the disease. The average treatment delay in our study was four years. Only $28 \%$ (85) of patients were treated within $>12$ months after diagnosis as treatment-naïve. Nearly $27 \%$ started the treatment later than five years, and $11 \%$ later than 10 years, after diagnosis. Despite not proven worsening of efficacy, treatment delay in our study was twice as long as that observed in a comparable time frame in Spain [28]. Taking into consideration the differences between treatment-naïve and retreated patients, we could only confirm a worse outcome in patients with profound clinical disability on treatment initiation, without differences between these two groups.

In previous Polish analysis, immunomodulatory drugs were used in only $24 \%$ of patients: mainly INF $\beta(81 \%)$ and GA (13\%) [29]. This time, the analysis showed a similar proportion and consistent INF $\beta$ dominance, in contrast to other countries [19]. In a similar observation period in the US, nearly $40 \%$ of newly-diagnosed MS patients were on treatment [30]. This shows that the problem with treatment initiation is not only related to country-specific regulations or budget, but also to patients' and physicians' beliefs in its efficacy and safety.

In a long term observation of injectable DMTs, the stop/ switch rates were mainly dependent on treatment duration, with intolerance as the primary reason for stopping, followed by inefficacy [19, 31-37]. Persistence in specific therapy indirectly describes the treatment efficacy. In our study, the efficacy was over $80 \%$ but the persistence was influenced rather by no other treatment options at that time.

Our study had some methodological shortcomings due to its multi-centre nature. Follow-up MRI scans were performed and analysed in different locations, albeit under one assessment protocol [38]. The same is true for EDSS scoring. An average treatment delay estimated as almost four years after MS diagnosis is another limitation of our study. However, this reflected the fact that country-specific qualification procedures led to late initiation of the treatment, which is inconsistent with common standards. We could determine the baseline predictive correlations only in univariate logistic regression analysis.

Many novel MS medications have been registered recently [39] and integrated to an improved MS treatment programme in Poland. Confronting the previously used algorithms with well-established outcomes control (RIO score) that we have used so far provides the insight that injectable DMTs are effective in RRMS patients (causing lowering of ARR and disease activity reflected in MRI) with limitation to less active and less clinically well patients. Our findings confirm that a careful baseline variables assessment can be helpful in predicting treatment response in injectable first-line drugs.

\section{Conclusions}

Patients with greater disability (EDSS $\geq 3$ ) at treatment initiation or hemiparesis as the initial symptom were more likely to fail at the second year of treatment. A high relapse rate one year prior to treatment adversely influences the treatment success at three years. Our findings confirm that first-line injectable drugs should not be chosen for treatment initiation in motoric disabled patients with a high grade of clinical activity. These drugs are effective in less active MS-RR patients.

Funding. This publication was prepared without any external sources of funding.

Conflict of interest. None declared.

Acknowledgement and financial support. None declared.

\section{References}

1. Brola W, Sobolewski P, Flaga S, et al. Increasing prevalence and incidence of multiple sclerosis in Poland. Neurologia i Neurochirurgia Polska. 2017; 51(1): 82-85, doi: 10.1016/j.pjnns.2016.11.005.

2. Rolak LA. Multiple sclerosis: it's not the disease you thought it was. Clin Med Res. 2003; 1(1): 57-60, indexed in Pubmed: 15931287.

3. Polman C, Reingold S, Edan G, et al. Diagnostic criteria for multiple sclerosis: 2005 revisions to the "McDonald Criteria". Annals of Neurology. 2005; 58(6): 840-846, doi: 10.1002/ana.20703.

4. Freedman MS, Selchen D, Arnold DL, et al. Canadian Multiple Sclerosis Working Group. Treatment optimization in MS: Canadian MS Working Group updated recommendations. Can J Neurol Sci. 2013; 40(3): 307-323, indexed in Pubmed: 23603165.

5. Interferon beta- $1 \mathrm{~b}$ is effective in relapsing-remitting multiple sclerosis: I. Clinical results of a multicenter, randomized, double-blind, placebocontrolled trial. Neurology. 1993; 43(4): 655-661, doi: 10.1212/ wnl.43.4.655.

6. Johnson KP, Brooks BR, Cohen JA, et al. Copolymer 1 reduces relapse rate and improves disability in relapsing-remitting multiple sclerosis: results of a phase III multicenter, double-blind placebo-controlled trial. 
The Copolymer 1 Multiple Sclerosis Study Group. Neurology. 1995; 45(7): 1268-1276, indexed in Pubmed: 7617181.

7. Jacobs LD, Cookfair DL, Rudick RA, et al. Intramuscular interferon beta-1a for disease progression in relapsing multiple sclerosis. The Multiple Sclerosis Collaborative Research Group (MSCRG). Ann Neurol. 1996; 39(3): 285-294, doi: 10.1002/ana.410390304, indexed in Pubmed: 8602746.

8. Randomised double-blind placebo-controlled study of interferon beta1a in relapsing/remitting multiple sclerosis. PRISMS (Prevention of Relapses and Disability by Interferon beta-1a Subcutaneously in Multiple Sclerosis) Study Group. Lancet. 1998; 352(9139): 1498-1504, indexed in Pubmed: 9820297.

9. Ziemssen T, Stefano NDe, Sormani M, et al. Optimizing therapy early in multiple sclerosis: An evidence-based view. Multiple Sclerosis and Related Disorders. 2015; 4(5): 460-469, doi: 10.1016/j. msard.2015.07.007.

10. Kurtzke JF. Rating neurologic impairment in multiple sclerosis: an expanded disability status scale (EDSS). Neurology. 1983; 33(11): 1444-1452, indexed in Pubmed: 6685237.

11. Sormani MP, Rio J, Tintorè $\mathrm{M}$, et al. Scoring treatment response in patients with relapsing multiple sclerosis. Multiple Sclerosis Journal. 2012; 19(5): 605-612, doi: 10.1177/1352458512460605.

12. Río J, Nos $\mathrm{C}$, Tintoré $\mathrm{M}$, et al. Defining the response to interferon-beta in relapsing-remitting multiple sclerosis patients. Ann Neurol. 2006; 59(2): 344-352, doi: 10.1002/ana.20740, indexed in Pubmed: 16437558.

13. Río J, Castilló J, Rovira A, et al. Measures in the first year of therapy predict the response to interferon $\beta$ in MS. Multiple Sclerosis Journal. 2009; 15(7): 848-853, doi: 10.1177/1352458509104591.

14. Rudick RA, Lee JC, Simon J, et al. Defining interferon beta response status in multiple sclerosis patients. Ann Neurol. 2004; 56(4): 548555, doi: 10.1002/ana.20224, indexed in Pubmed: 15389896.

15. Río J, Rovira À, Tintoré M, et al. Relationship between MRI lesion activity and response to IFN- $\beta$ in relapsing-remitting multiple sclerosis patients. Multiple Sclerosis Journal. 2008; 14(4): 479-484, doi: 10.1177/1352458507085555.

16. Río J, Comabella M, Montalban X. Predicting responders to therapies for multiple sclerosis. Nature Reviews Neurology. 2009; 5(10): 553-560, doi: 10.1038/nrneurol.2009.139.

17. Havrdova E, Galetta S, Stefoski D, et al. Freedom from disease activity in multiple sclerosis. Neurology. 2010; 74(Issue 17, Supplement 3): S3-S7, doi: 10.1212/wnl.0b013e3181dbb51c.

18. Hyun JW, Kim SH, Jeong I, et al. Utility of the Rio Score and Modified Rio Score in Korean Patients with Multiple Sclerosis. PLOS ONE. 2015; 10(5): e0129243, doi: 10.1371/journal.pone.0129243.

19. Zhornitsky S, Greenfield J, Koch M, et al. Long-Term Persistence with Injectable Therapy in Relapsing-Remitting Multiple Sclerosis: An 18Year Observational Cohort Study. PLOS ONE. 2015; 10(4): e0123824, doi: 10.1371/journal.pone.0123824.

20. Oleen-Burkey M, Cyhaniuk A, Swallow E. Retrospective US database analysis of persistence with glatiramer acetate vs. available diseasemodifying therapies for multiple sclerosis: 2001-2010. BMC Neurology. 2014; 14(1), doi: 10.1186/1471-2377-14-11.

21. Mesaroš Š, Stojsavljević N, Dujmović-Bašuroski I, et al. Long-term adherence to interferon-beta treatment in a cohort of RRMS patients in Belgrade, Serbia. Clinical Neurology and Neurosurgery. 2012; 114(8): 1145-1148, doi: 10.1016/j.clineuro.2012.02.043.
22. Limmroth V, Putzki N, Kachuck NJ. The interferon beta therapies for treatment of relapsing-remitting multiple sclerosis: are they equally efficacious? A comparative review of open-label studies evaluating the efficacy, safety, or dosing of different interferon beta formulations alone or in combination. Ther Adv Neurol Disord. 2011; 4(5): 281-296, doi: 10.1177/1756285611413825, indexed in Pubmed: 22010041.

23. Khan OA, Tselis AC, Kamholz JA, et al. A prospective, open-label treatment trial to compare the effect of IFNbeta-1a (Avonex), IFNbeta- $1 \mathrm{~b}$ (Betaseron), and glatiramer acetate (Copaxone) on the relapse rate in relapsing-remitting multiple sclerosis: results after 18 months of therapy. Mult Scler. 2001; 7(6): 349-353, doi: 10.1177/135245850100700601, indexed in Pubmed: 11795454.

24. Sorensen PS, Koch-Henriksen N, Ravnborg M, et al. Danish Multiple Sclerosis Study Group. Immunomodulatory treatment of multiple sclerosis in denmark: a prospective nationwide survey. Mult Scler. 2006; 12(3): 253-264, doi: 10.1191/135248506ms1323oa, indexed in Pubmed: 16764337.

25. Hass J, Firzlaff M. Twenty-four-month comparison of immunomodulatory treatments - a retrospective open label study in 308 RRMS patients treated with beta interferons or glatiramer acetate (Copaxone). Eur J Neurol. 2005; 12(11): 916-917.

26. Río J, Porcel J, Téllez N, et al. Factors related with treatment adherence to interferon beta and glatiramer acetate therapy in multiple sclerosis. Mult Scler. 2005; 11(3): 306-309, doi: 10.1191/1352458505ms1173oa, indexed in Pubmed: 15957512.

27. Cunningham A, Gottberg K, von Koch L, et al. Non-adherence to interferon-beta therapy in Swedish patients with multiple sclerosis. Acta Neurol Scand. 2010; 121(3): 154-160, doi: 10.1111/j.1600-0404.2009.01285.x, indexed in Pubmed: 20055771.

28. Fernández 0 , Fernández V, Arbizu T, et al. Novo Group. Characteristics of multiple sclerosis at onset and delay of diagnosis and treatment in Spain (the Novo Study). J Neurol. 2010; 257(9): 1500-1507, doi: 10.1007/s00415-010-5560-1, indexed in Pubmed: 20383518.

29. Kułakowska A, Bartosik-Psujek H, Hożejowski R, et al. Selected aspects of the epidemiology of multiple sclerosis in Poland - a multicentre pilot study. Neurol Neurochir Pol. 2010; 44(5): 443-452, indexed in Pubmed: 21082490.

30. Margolis J, Fowler R, Johnson B, et al. Disease-modifying drug initiation patterns in commercially insured multiple sclerosis patients: a retrospective cohort study. BMC Neurology. 2011; 11(1), doi: 10.1186/1471-2377-11-122.

31. Wong J, Gomes T, Mamdani M, et al. Adherence to multiple sclerosis disease-modifying therapies in Ontario is low. Can J Neurol Sci. 2011; 38(3): 429-433, indexed in Pubmed: 21515501.

32. Pittock SJ, Mayr WT, McClelland RL, et al. Disability profile of MS did not change over 10 years in a population-based prevalence cohort. Neurology. 2004; 62(4): 601-606, indexed in Pubmed: 14981177.

33. Limmroth V, Malessa R, ZettI UK, et al. QUASIMS Study Group. Quality Assessment in Multiple Sclerosis Therapy (QUASIMS): a comparison of interferon beta therapies for relapsing-remitting multiple sclerosis. J Neurol. 2007; 254(1): 67-77, doi: 10.1007/s00415-006-0281-1, indexed in Pubmed: 17273808.

34. Pittock SJ, Mayr WT, McClelland RL, et al. Disability profile of MS did not change over 10 years in a population-based prevalence cohort. Neurology. 2004; 62(4): 601-606, indexed in Pubmed: 14981177. 
35. Limmroth V, Malessa R, ZettI UK, et al. QUASIMS Study Group. Quality Assessment in Multiple Sclerosis Therapy (QUASIMS): a comparison of interferon beta therapies for relapsing-remitting multiple sclerosis. J Neurol. 2007; 254(1): 67-77, doi: 10.1007/s00415-006-0281-1, indexed in Pubmed: 17273808.

36. Mikol DD, Barkhof F, Chang P, et al. REGARD study group. Comparison of subcutaneous interferon beta-1a with glatiramer acetate in patients with relapsing multiple sclerosis (the REbif vs Glatiramer Acetate in Relapsing MS Disease [REGARD] study): a multicentre, randomised, parallel, open-label trial. Lancet Neurol. 2008; 7(10): 903-914, doi: 10.1016/S1474-4422(08)70200-X, indexed in Pubmed: 18789766.
37. Jokubaitis V, Spelman T, Lechner-Scott J, et al. The Australian Multiple Sclerosis (MS) Immunotherapy Study: A Prospective, Multicentre Study of Drug Utilisation Using the MSBase Platform. PLoS ONE. 2013; 8(3): e59694, doi: 10.1371/journal.pone.0059694.

38. Hartel M, Kluczewska E, Pierzchała K, et al. What you cannot get from routine MRI of MS patient and why - The growing need for atrophy assessment and seeing beyond the plaque. Neurol Neurochir Pol. 2016; 50(2): 123-130, doi: 10.1016/j.pjnns.2016.01.007, indexed in Pubmed: 26969569.

39. Wingerchuk D, Carter J. Multiple Sclerosis: Current and Emerging Disease-Modifying Therapies and Treatment Strategies. Mayo Clinic Proceedings. 2014; 89(2): 225-240, doi: 10.1016/j.mayocp.2013.11.002. 